\title{
Modelling HIV Intervention among Most-at-Risk/Key Population: Case Study of FWSS in Nigeria
}

\author{
Sampson Akwafuo*, Andrew Shattock and Armin R Mikler
}

University of North Texas, Denton, TX, USA

\begin{abstract}
Using FWSS in Nigeria as a case study, this research develops a novel risk equation for estimating new infections among FWSS, their clients and communities. It uses a hybrid SUDT and SIT structural model. It considers number of contacts, number of protected and unprotected sexual acts, population and other existing values as base inputs. Simulation of the model was done using python programming. The model also estimates the impacts of these interventions on the clients of the sex workers, their female partners and the general population. The levels of the program implementation, needed on each scenario, to achieve the required number of averted new infections are also modelled. This model can be used to estimate the risk of a population set to a sexually transmitted disease. Public health workers can use the model to prepare a fit for- purpose intervention program for specific community members.
\end{abstract}

Keywords: FWSS; FSW; MARPs; Simulation; Mathematical modelling; Python programming

\section{Introduction}

Nigeria, with a population of 186 Million (2016 estimate), is the largest country in Africa. With over 3.5 million HIV positive persons, Nigeria has the second highest HIV burden globally. An estimated $60 \%$ of new HIV infections in Western and Central Africa in 2015 occurred in Nigeria [1]. Men who have Sex with Men (MSM), Female who Sell Sex (FWSS) and People who Injects Drugs (PWID) account for about 32\% of new HIV infections in Nigeria and are the worst affected population sets by the epidemic. FWSS account for $20 \%$ of new infections in Nigeria. The country level prevalence for Brothel-Based Female who Sell Sex (BBFWSS) was 19.4\% in 2014

Using FWSS in Nigeria as a case study, this research develops a novel risk equation for estimating new infections among FWSS, their clients and communities. It uses a hybrid SUDT and SIT structural model. It considers number of contacts, number of protected and unprotected sexual acts, population and other existing values as base inputs. Simulation of the model was done using python programming. The model also estimates the impacts of these interventions on the clients of the sex workers, their female partners and the general population. The levels of the programme implementation, needed on each scenario, to achieve the required number of averted new infections are also modelled. This model can be used to estimate the risk of a population set to a sexually transmitted disease. Public health workers can use the model to prepare a fit-for-purpose intervention program for specific community members.

\section{Objective}

The objective of this research is to develop a mathematical model to estimate how many indirect HIV infections will be averted among FWSS, their clients and the general population, attributable to prevention programs targeting female sex works in Nigeria. The model also estimates the impacts of these interventions on the clients of the sex workers, their female partners and the general population. The model includes a risk ratio used to estimate the impact of the programme on each of the sub-population sets. The number of new infections averted on the sex workers and their clients, attributable to different scenarios and levels of the programme implementation is presented in this paper.

\section{Methodology}

A mathematical model was developed, using python programming language. A hybrid S-U-D-T and S-I-T structures were used in designing the model. The I group of FWSS was further divided into $\mathrm{U}$ and D while S-I-T was used for other population groups. A Susceptible (S) group includes all members of the population set; The Undiagnosed (U) group includes infected members of the population that are yet to be tested. The Diagnosed are the tested and confirmed members of the population set. The Infected (I) group includes both tested and yet-tobe tested infected persons $(\mathrm{I}=\mathrm{U}+\mathrm{D})$; The Treatment group involves all persons on treatment and care [2].

The model considers various factors affecting the implementations of the program. Current values of the model variables, as shown in the Table 1 below, served as baseline inputs to the model [3]. The variables include initial prevalence of HIV among FWSS, their clients; proportion of sex acts that are protected; initial population of the target group; duration of the intervention; number of sexual contacts per FWSS, average number of sexual acts per week, etc. A specific risk equation was developed for the FSW, incorporating the current value of each variable [4]. Three Scenarios of the model was estimated over a period of five years. The first scenario entails putting all infected FWSS on treatment, irrespective of their CD4 or WHO staging and keeping other variables constant. The model considers the impact of this scenario on the clients of the sex workers. Putting only eligible FSWs on treatment and increasing condom distribution was the second scenario. Universal access to treatment for all FSWs and their clients was then modelled. An uncertainty analysis was also carried out as part of the model [5] (Figures 1-7).

*Corresponding author: Sampson Akwafuo, University of North Texas, Denton TX, United States, Tel: 4694516075; E-mail: sampson_akwa@yahoo.co.uk

Received September 10, 2017; Accepted September 21, 2017; Published September 28, 2017

Citation: Akwafuo S, Shattock A, Mikler AR (2017) Modelling HIV Intervention among Most-at-Risk/Key Population: Case Study of FWSS in Nigeria. J AIDS Clin Res 8: 732. doi: 10.4172/2155-6113.1000732

Copyright: (c) 2017 Akwafuo S, et al. This is an open-access article distributed under the terms of the Creative Commons Attribution License, which permits unrestricted use, distribution, and reproduction in any medium, provided the original author and source are credited. 
Citation: Akwafuo S, Shattock A, Mikler AR (2017) Modelling HIV Intervention among Most-at-Risk/Key Population: Case Study of FWSS in Nigeria. J AIDS Clin Res 8: 732. doi: 10.4172/2155-6113.1000732

Page 2 of 4

\begin{tabular}{|c|c|c|c|}
\hline Parameters & Description & Symbol & Value \\
\hline (FWSS) & Proportion of FWSS that are infected & $p_{s w}$ & 19.5 \\
\hline Initial prevalence in GF & Proportion of Gen Female that are infected & $p_{G F}$ & 4.1 \\
\hline Initial prevalence in GM & Proportion of Gen Male that are infected & $\mathrm{p}_{\mathrm{GM}}$ & 3.2 \\
\hline Efficacy of condom & Efficacy of condom & $\varepsilon$ & 0.95 \\
\hline Proportion of FWSS that are infected with STI & Proportion of FWSS that are infected with STI & $\mathrm{p}_{\mathrm{sti}}$ & 28.5 \\
\hline Maturity rate & Rate of maturity into adulthood for gen population & $\mathrm{m}$ & 15 \\
\hline No of acts 1 & Number of acts in FWSS-Clients & $\mathrm{n}_{1}$ & 200 \\
\hline No of acts 2 & Number of acts in Gen Population & $\mathrm{n}_{2}$ & 80 \\
\hline No of contacts 1 & Number of contacts of the FWSS & c1 & 432 \\
\hline No of contacts 2 & Number of contacts of the Clients & c2 & 50 \\
\hline No of contacts 3 & Number of contacts of the GF, GM & c3 & 2 \\
\hline Beta & Rate of transmission (general unprotected sex) & ß & 0.01 \\
\hline Initial Population of GM & Initial male population size & NGM & $39,208,214$ \\
\hline Initial Population of FSW & Initial FSW population size & NSW & 308340 \\
\hline Initial Population of Clients & Initial Clients population size & $\mathrm{NCL}$ & 18500400 \\
\hline Initial Population of GF & Initial Female population size & NGF & 38942286 \\
\hline Death rate among susceptible & Natural death rate among susceptible FWSS, GF, CL \& GM & $\mathrm{d}$ & 0.014 \\
\hline Death rate among infected & Death rate among infected FWSS, GF, CL \& GM & $\mu \mathrm{HIV}$ & 0.12 \\
\hline Death rate among those treatment & Death rate among FWSS, GF, CL \& GM on treatment & $\mu \mathrm{T}$ & 0.03 \\
\hline Tethar FSW & Rate of migration from Undiagnosed to Diagnosed FWSS & $\psi_{\mathrm{sw}}$ & 0.6 \\
\hline Tethar Treatment FSW & Rate of migration from diagnosed to treatment FSW & ФSW & 0.05 \\
\hline Tethar Treatment & Rate of migration from infected to treatment & $\Phi \mathrm{T}$ & 0.33 \\
\hline Rate of migration/upwards zero & Rate of migration from SGF to SFSW; and IGF to UFSW & go & 1 \\
\hline Rate of migration/downwards zero & Rate of migration from SFSW and UFSW to SGF \& IGF & ho & 1 \\
\hline Rate of migration/upwards one & Rate of migration from IGF \& TGF to DFSW and TFSW & g1 & 1 \\
\hline Rate of migration/downwards one & Rate of migration from DFSW and TFSW to IGF \& TGF & h1 & 1 \\
\hline Rate of migration (Gen Male) & Rate of migration from $\mathrm{GM}$ to Clients & g2 & 1 \\
\hline Rate of migration (Gen Male) & Rate of migration from Clients to GM & h2 & 1 \\
\hline Rate of migration/upwards zero & Rate of migration from SGF to SFSW; and IGF to UFSW & go & 1 \\
\hline Rate of migration/downwards zero & Rate of migration from SFSW and UFSW to SGF \& IGF & ho & 1 \\
\hline Rate of migration/upwards one & Rate of migration from IGF \& TGF to DFSW and TFSW & g1 & 1 \\
\hline Rate of migration/downwards one & Rate of migration from DFSW and TFSW to IGF \& TGF & h1 & 1 \\
\hline Rate of migration (Gen Male) & Rate of migration from GM to Clients & g2 & 1 \\
\hline Rate of migration (Gen Male) & Rate of migration from Clients to GM & h2 & 1 \\
\hline
\end{tabular}

Table 1: Parameters, description, symbol and values used.

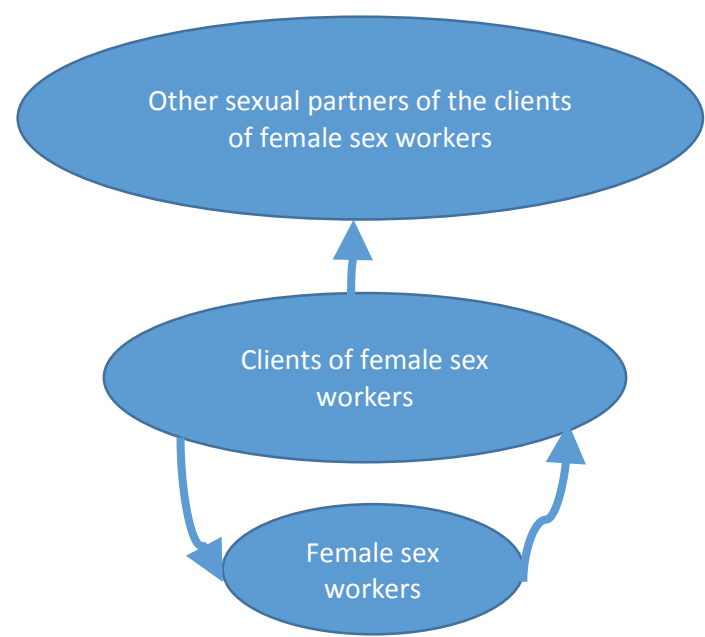

Figure 1: Schematic diagram of the FWSS sexual network. The size of the circles represents the relative size of the sub-populations. The arrows represent the predominant direction of HIV infection from infected people to uninfected people.

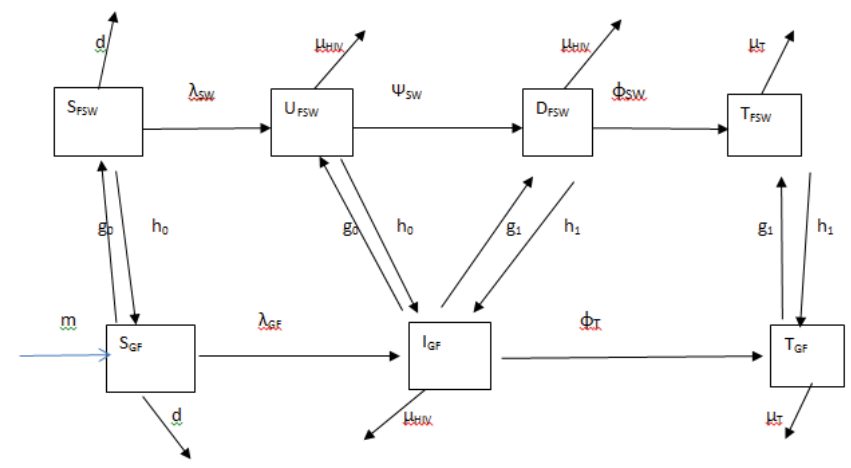

Figure 2: Interaction and model structure of female who sell sex (formerly known as FSW) and the general female (GF).

\section{Assumptions}

- Net rate of migration from SFWSS group to SGF is the same as SGF>>SFWSS, (go/ho), UFSW and different from IGF to DFSW and TFSW 
- There are different rate of migration for each of the sub-groups

- There are different death rate for Susc, Und, Diag, Infected and Treatment group

- No significant sexual contact between FSW and GF

- Individuals in the infected population, not on ART, lives for extra 10 to 15 years

- Maturity rate of 15 years

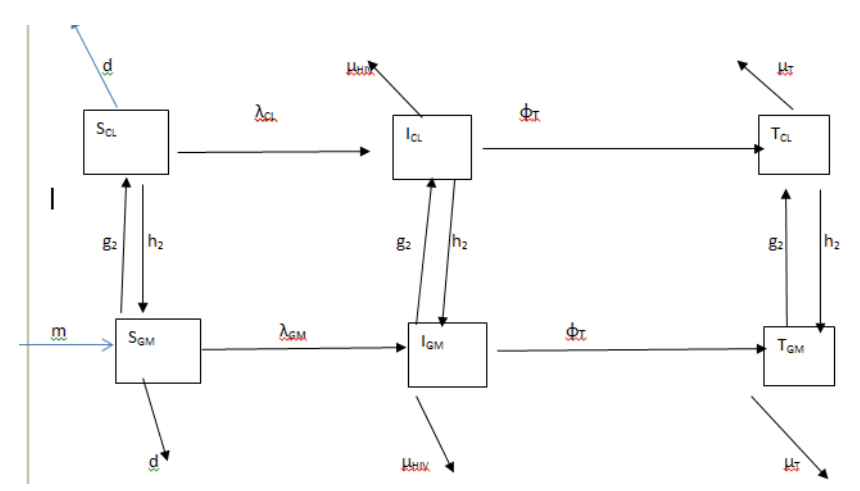

Figure 3: Interaction and model structure of the clients $(\mathrm{CL})$ and general male (GM).

- Only GF within the age range of 15-49 are considered

\section{Modelling Equations}

\section{Equations for FSW}

$$
\begin{aligned}
& S(t+1)=S_{F S W}(t)+g_{o} S_{G F}(t)-\lambda_{s w} S_{F S W}(t)-d S_{F S W}(t)-h_{o} S_{F S W}(t) \\
& U(t+1)=U(t)+\lambda_{s w} . S(t)-\mu_{H I V} U(t)-\Psi_{f s w} U(t)+g_{o} I_{G F}(t)-h_{o} U(t) \\
& D(t+1)=D(t)+\Psi_{f s w} U(t)-\mu_{H I V} D(t)-\phi_{f s w} D(t)+g_{1} I_{G F}(t)-h_{1} D(t) \\
& T(t+1)=T_{f s w}(t)+\phi_{f s w} D(t)-\mu_{T} T_{f S w}(t)+g_{1} T_{G F}(t)-h_{1} T_{f s w}(t)
\end{aligned}
$$

\section{Risk equation for FSW}

$$
\begin{aligned}
& \lambda_{S W}=\left[C _ { 1 } * \frac { I _ { C L } } { N _ { C L } } * ( 1 - P _ { S i } ) * \left\{\left(1-(1-\beta)^{n(1-p)}\right\}\left\{\left(1-((1-\varepsilon) \beta)^{n p}\right\}\right]+\left[C_{1} * I_{C L} / N_{C L} * P s t i\right.\right.\right. \\
& \left\{\left(1-\left(e_{s i t} \beta\right)^{n(1-p)}\right\}\left\{\left(1-\left((1-\varepsilon) e_{s i t} \beta\right)^{n p}\right\}\right]+\left[c_{1} * \frac{T_{C L}}{N_{C L}} *\left(1-p_{s i t}\right)\right.\right. \\
& \left.*\left\{\left(1-\left(\left(1-\varepsilon_{T}\right) \beta\right)\right\}^{n(1-p)}\right\}\left\{\left(1-\left(1-\varepsilon_{T}\right)(1-\varepsilon) \beta\right)^{n p}\right\}\right]+ \\
& {\left[C_{1} * P_{s i t} * T_{C L} / N_{C L} *\{(1-(1-\right.} \\
& \left.\left.\left.\left.\left(1-\varepsilon_{T}\right) e_{s s i} \beta\right)\right\}\right\}^{n(1-p)} *\left\{\left(1-\left(1-\varepsilon_{T}\right)(1-\varepsilon) e_{s i t} \beta\right)^{n p}\right\}\right]
\end{aligned}
$$

\section{Equation for general female}

$$
\begin{aligned}
& S(t+1)=S_{G F}(\mathrm{t})+M_{1} S_{G F}(\mathrm{t})-g_{o} S_{G F}(t)+h_{o} S_{F S W}(t)-\lambda_{G F}(t) S_{G F}(t)-d S_{G F}(t) \\
& I(t+1)=I_{G F}(t)+\lambda_{G F} S_{G F}(\mathrm{t})+h_{o} U(t)-g_{o} I_{G F}(t)-g_{1} I_{G F}(t)+h_{1} D(t)-\phi_{T} I_{G F}(t)-\mu_{H I V} I_{G F}(t) \\
& T(t+1)=T_{G F}(t)+\phi_{T} I_{G F}(t)-g_{1} T_{G F}(t)+h_{1} T_{f s w}(t)-\mu_{T} T_{G F}(t)
\end{aligned}
$$

Treated CL
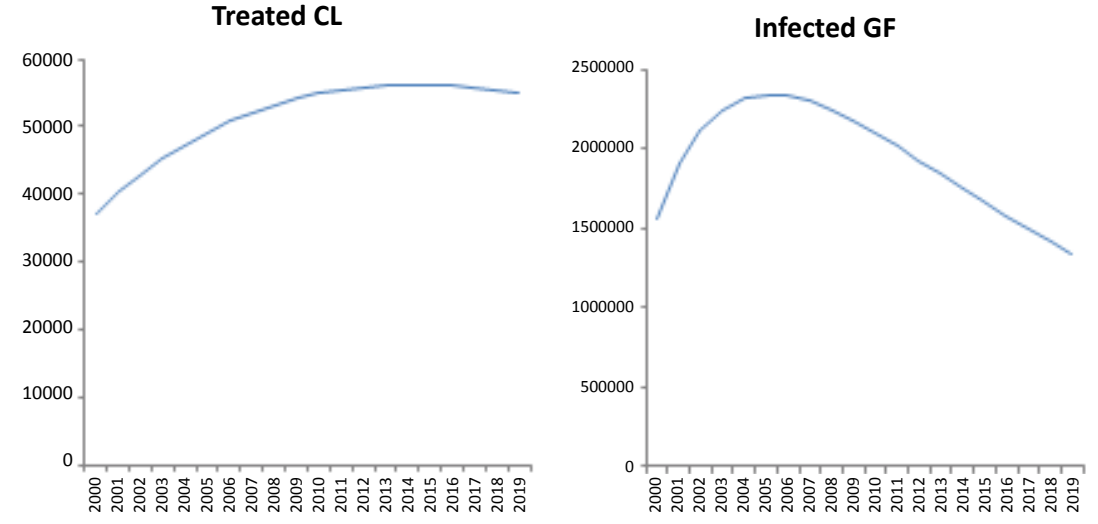

Figure 4: Impacts of treatment of clients on the general population.

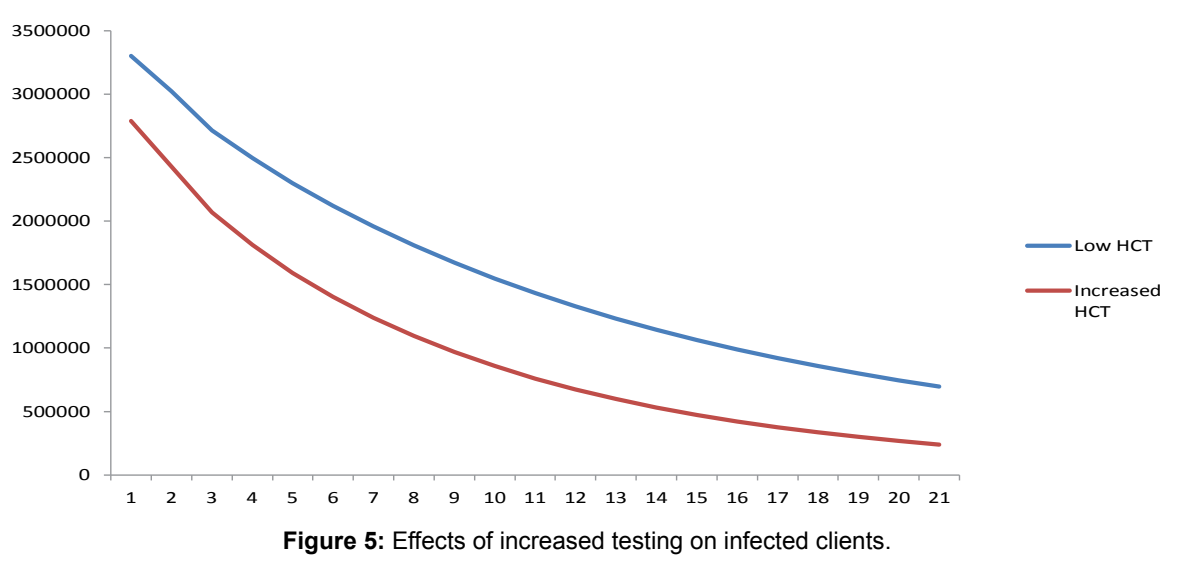




\section{Treated FSW}

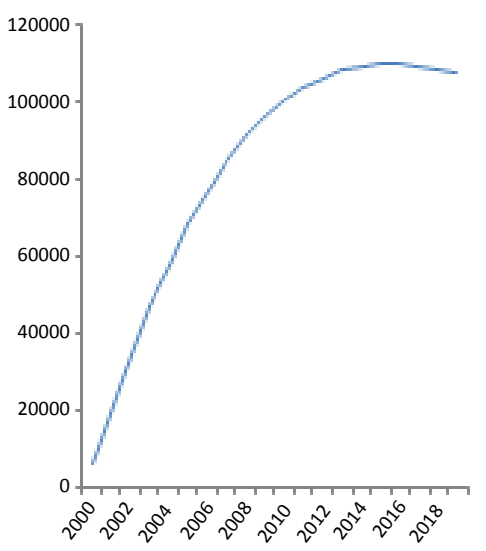

\section{Infected GM}

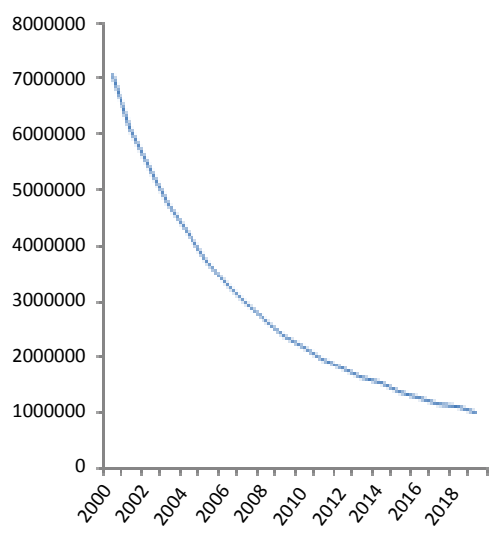

Figure 6: Impacts of treatment of FSW on the general population.

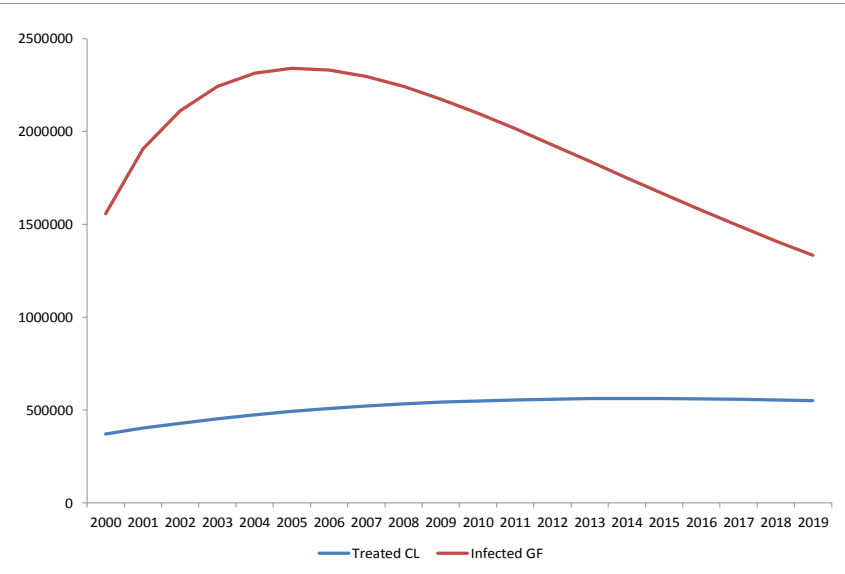

Figure 7: Increase in the treatment of clients reduces infection in general female.

Risk equation for general female

$$
\begin{aligned}
& \lambda_{G F}=\left[C_{2} * \frac{I_{C L}}{N_{C L}} *\left\{\left(1-(1-\beta)^{n \cdot(1-p)}\right\}\left(1-(1-\varepsilon) \beta^{n p}\right)\right]+\left[C_{2} * T_{C L} / N_{C L}\right.\right. \\
& \left.\left.*\left(1-\left(1-\left(1-\varepsilon_{T}\right) \beta\right)^{n(1-p)}\right)\left(1-\left(1-\varepsilon_{T}\right)(1-\varepsilon) \beta\right)^{n p}\right)\right]+ \\
& \left.\left[C_{2} * I_{G M} / N_{G M} *\left(1-(1-\beta)^{n(1-p)}\right)(1-(1-\varepsilon) \beta)^{n p}\right)\right]+ \\
& {\left[C_{2} * T_{G M} / N_{G M} *\right.} \\
& \left.\left.\left(1-\left(1-\left(1-\varepsilon_{T}\right) \beta\right)^{n(1-p)}\right)\left(1-\left(1-\varepsilon_{T}\right)(1-\varepsilon) \beta\right)^{n p}\right)\right]
\end{aligned}
$$

\section{Client equation}

$$
\begin{aligned}
& S(t+1)=S_{C L}(t)-d S_{C L}(t)-\lambda_{C L}(t) . S_{C L}(t)-h_{2} S_{C L}(t)+g_{2} S_{G M}(t) \\
& I_{(t+1)}=I_{C L}(t)+\lambda_{C L}(t) S_{C L}(t)-\mu_{H I V} I_{C L}(t)-\phi_{T} I_{C L}+g_{2} I_{G M}(t)-h_{2} I_{C L}(t) \\
& T_{(t+1)}=T_{C L}(t)+\phi_{T} I_{C L}(t)-\mu_{T} T_{C L}(t)+g_{2} T_{C L}(t)-h_{2} T_{f s w}(t)
\end{aligned}
$$

\section{Client risk equation}

$$
\begin{aligned}
& \lambda_{C L}=\left[C_{2} * I_{G F} / N_{G F} *\left(1-(1-\beta)^{n(1-p)}\right)\left(1-((1-\varepsilon) \beta)^{n p}\right]+\right. \\
& {\left[C_{2} * T_{G F} / N_{G F} *\left(1-\left(\left(1-\left(1-\varepsilon_{T}\right) \beta\right)^{n(1-p)}\right)\left(1-\left(1-\varepsilon_{T}\right)(1-\varepsilon) \beta\right)^{n p}\right)\right]} \\
& +\left[C_{1} * I_{F S W} / N_{F S W} *\left(1-(1-\beta)^{n(1-p)}(1-(1-\varepsilon) \beta)^{n p}\right)\right] \\
& +\left[C_{1} * T_{F S W} / N_{F S W} *\left(1-\left(1-\left(1-\varepsilon_{T}\right) \beta\right)^{n(1-p)}\left(1-\left(1-\varepsilon_{T}\right)(1-\varepsilon) \beta\right)^{n p}\right]\right.
\end{aligned}
$$

\section{General male equation}

$$
\begin{aligned}
& S(t+1)=S_{G M}(t)+M_{1} S_{G M}(\mathrm{t})-g_{2} S_{G M}(t)+h_{2} S_{C L}(t)-\lambda_{G M}(t) S_{G M}(t)-d S_{G M}(t) \\
& I_{(t+1)}=I_{G M}(t)+\lambda_{G M}(t) \cdot S_{G M}(t)+h_{2} I_{C L}(t)-g_{2} I_{G M}(t)-\phi_{T} I_{G M}(t)-\mu_{H V} I_{G M}(t) \\
& T_{(t+1)}=T_{G M}(t)+\phi_{T} I_{G M}(t)-g_{2} T_{G M}(t)+h_{2} T_{C L}(t)-\mu_{T} T_{G M}(t)
\end{aligned}
$$

\section{General male risk equation}

$$
\begin{aligned}
& \lambda_{G M}=\left[C_{2} * I_{G F} / N_{G F} *\left(1-(1-\beta)^{n(1-p)}\left((1-(1-\varepsilon) \beta)^{n p}\right]+\right.\right. \\
& {\left[C_{2} * T_{G F} / N_{G F} *\left(1-\left(1-\left(1-\varepsilon_{T}\right) \beta\right)^{n(1-p)}\right)\left(1-\left(\left(1-\varepsilon_{T}\right)(1-\varepsilon) \beta\right)^{n p}\right]\right.}
\end{aligned}
$$

\section{Results and Discussion}

It was observed that if the status quo (37\% of eligible positive FSW on treatment) is maintained, the new infection rate will gradually increase by $3.6 \%$ in five years' time. Putting $80 \%$ of eligible positive FSWs on treatment will avert 2789 new infections in the same duration and reduce the current rate of new infections to 0.7 . A slight decrease of $0.3 \%$ would be experienced in the general female population. Putting all FSWs on treatment returns a $89.7 \%$ reduction on the number of new infections among clients of FSW.

\section{Conclusion and Recommendations}

The simulation model reveals the efficiency of treatment in reducing the rate of new infections among FSWs, their clients and general female. The models reveal the importance of the investing in the FSW intervention programs now, rather in the future. The model outputs can be used to calculate the Quality Adjusted Life Years (QALY) to be gained during the intervention. A slight contribution of the total number of condom distributed to a reduction in new infection rate was also noticed. Further modelling scenarios are required to effectively infer on the efficiency of the intervention programs.

\section{References}

1. Global Information and Education on HIV and AIDS (2016) Avert Publications, Nigeria.

2. Hernandez-Vargasa EA, Middleton RH (2013) Modelling the three stages in HIV infection. J Theor Biol 320: 33-40.

3. Perelson AS, Ribeiro RM (2013) Modelling the within-host dynamics of HIV infection. BMC Biol 11: 96

4. Wodars D, Cassels S (2012) Mathematical models of HIV pathogenesis and treatment. Harvard Publications.

5. Alizon S, Magnus C (2012) Modelling the course of an HIV infection: Insights from ecology and evolution. Viruses 4: 1984-2013. 